\title{
Modelling Based Estimates for Severe Pneumonia and Pneumonia Deaths in Indian States
}

\author{
Habib H. Farooqui¹, David Heymann² and Sanjay Zodpey ${ }^{1}$ \\ ${ }^{1}$ Public Health Foundation of India, Gurgaon, India; ${ }^{2}$ London School of Hygiene and Tropical Medicine, London, United Kingdom
}

\section{Objective}

This presentation highlights the use of mathematical model to estimate burden of disease in absence surveillance data. We estimated the burden of severe pneumonia, pneumococcal pneumonia and pneumonia deaths in Indian states using a mathematical model through application of vaccine probe methodology and attributable fraction.

\section{Introduction}

The Child Health Epidemiology Reference Group (CHERG) has predicted around 43 million pneumonia cases in India. It is recognized that for huge nation like India, which accounts for $23 \%$ of global pneumonia burden, the national estimates may hide regional disparities(1). In this context, we have generated Indian state specific burden of severe pneumonia, pneumococcal pneumonia and pneumonia deaths through use of mathematical model.

\section{Methods}

We developed a Microsoft Excel-based model to estimate number of new episodes of severe pneumonia for each Indian state. This model is based on the epidemiological concept of potential impact fraction(1) as follows:

$\mathrm{N}_{\mathrm{e} / \mathrm{cy}}=\left(\mathrm{Pop}_{<\mathrm{lyrs}}\right) \mathrm{x}\left(\mathrm{Inc}_{\mathrm{Ind}}\right) \mathrm{x}\left\{1+\Sigma_{(\mathrm{RF}=1->\mathrm{n})}\left[\left(\mathrm{Prev}_{\mathrm{RFn}}-\operatorname{Prev}_{\mathrm{RFnInd}}\right) \mathrm{X}\right.\right.$ $\left.\left.\left(\mathrm{RR}_{\mathrm{RFn}}-1\right)\right]\right\}$,

where $\mathrm{Ne} / \mathrm{cy}$ is the number of new episodes of clinical pneumonia per year in selected Indian state, Pop ${ }_{<\text {yrs }}$ is the population of children less than 1 years in each state, $\mathrm{Inc}_{\text {Ind }}$ is the estimated incidence of severe clinical pneumonia at all India level, $\operatorname{Prev}_{\mathrm{RFn}}$ is the prevalence of exposure to $\mathrm{n}$-th risk factor among under-fives in the Indian state of interest, Prev $\mathrm{RFnInd}_{\mathrm{i}}$ is the prevalence of exposure to $\mathrm{n}$-th risk factor among under-fives at all India level and $\mathrm{RR}_{\mathrm{RFn}}$ is the relative risk for developing clinical pneumonia associated with the $\mathrm{n}$-th risk factor. We then estimated the number of pneumococcal pneumonia cases by applying the vaccine probe methodology to an existing Philippines trial. The study reported $19.8 \%$ efficacy against radiologic pneumonia ((95\% CI: $-8.8,40.8)$ in children age less than 1 year(2). The 11 serotypes contained in the vaccine were estimated to account for $65.33 \%$ of IPD in India. With vaccine efficacy against vaccinetype pneumococcal disease of $83 \%$ (3), we observed that $23.8 \%$ of radiologic pneumonia cases are due to vaccine serotypes and $36.51 \%$ due to any pneumococcal serotypes. The mortality rate in hospitalized cases of severe pneumonia (1.95\%) and pneumococcal pneumonia $(16.7 \%)$ in children age less than 5 years was estimated from multicentric studies.

\section{Results}

We estimated that in 2010, 3.57 million severe pneumonia cases and 0.35 million all cause pneumonia deaths occurred in children age less than 5 years in India. Three states requires specific mention, Uttar Pradesh contributed (24\% of severe pneumonia cases and $26 \%$ of pneumonia deaths), Bihar (16\% cases, $22 \%$ deaths) and MP ( $9 \%$ cases, $12 \%$ deaths) to the national figures. They were also top contributors pneumococcal pneumonia burden, i.e. UP $(1,33,160$ cases, 27,285 deaths), Bihar (91,574 cases, 23,202 deaths) and
MP (52,247 cases, 13,043 deaths). The total numbers of severe pneumococcal pneumonia cases and deaths in 2010 were estimated to be 0.56 million and 95 thousand respectively. The contribution of pneumococcal pneumonia was $15.8 \%$ to all cause pneumonia cases and $20.8 \%$ to all cause pneumonia deaths. The In age specific analysis, we observed that pneumonia related morbidity was highest in $0-1$ year age group (51\%) followed by 1-2 age group (22\%), 2-3 years $(11 \%), 3-4$ years $(9 \%)$ and $4-5$ years $(7 \%)$.

\section{Conclusions}

To summarize, the state-specific estimates are key for identification of states with high burden of pneumonia related morbidity and mortality and to target interventions for pneumonia prevention and control especially pneumococcal conjugate vaccines to achieve maximum impact.

\section{Keywords}

Mathematical Modelling; Pneumonia; Vaccine Probe; Pneumococcal Conjugate Vaccine; Pneumococcal Pneumonia

\section{Acknowledgments}

This work was supported by a Wellcome Trust Capacity Strengthening Strategic Award to the Public Health Foundation of India and a consortium of UK universities.

\section{References}

1. Rudan I, Boschi-Pinto C, Biloglav Z, Mulholland K, Campbell H. Epidemiology and etiology of childhood pneumonia. Bull World Health Organ. 2008;86(5):408-16.

2. Lucero MG, Nohynek H, Williams G, Tallo V, Simoes EA, Lupisan $S$, et al. Efficacy of an 11-valent pneumococcal conjugate vaccine against radiologically confirmed pneumonia among children less than 2 years of age in the Philippines: a randomized, double-blind, placebo-controlled trial. The Pediatric infectious disease journal. 2009;28(6):455-62.

3. Klugman KP, Madhi SA, Huebner RE, Kohberger R, Mbelle N, Pierce $\mathrm{N}$, et al. A trial of a 9-valent pneumococcal conjugate vaccine in children with and those without HIV infection. The New England journal of medicine. 2003;349(14):1341-8.

\section{*Habib H. Farooqui}

E-mail: drhabibhasan@gmail.com 